\title{
The root of all evil
}

Two studies of tumor ancestry reveal evidence for discrete populations of cancer stem cells.

Is cancer an 'equal-opportunity employer', or is it fueled by propagation of an 'elite' population of cancer stem cells (CSCs)? Amid considerable debate, the CSC hypothesis is steadily gaining experimental support.

"The prevailing notion has always been that essentially any cell can give rise to a tumor, but we don't believe that to be the case," says Luis Parada, of the University of Texas Southwestern Medical Center. Most CSC studies to date have involved transplantation, with the potential for artifacts arising from mov-

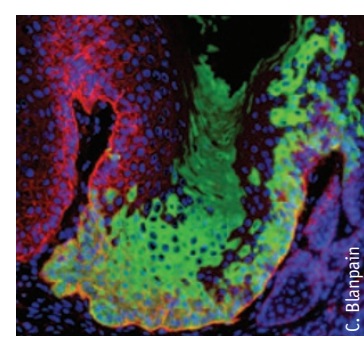

Within weeks, descendents of a single cell (green) can dominate a mouse papilloma. ing tumor cells from their native niche to a new location, but Parada's team and a group led by Cédric Blanpain at the Université Libre de Bruxelles have employed a more naturalistic approach to investigate tumor cells in their native environment.

"These experiments aren't simply looking at what cells can do," says Blanpain. "You're looking at what the cells really do." His team induced benign skin tumors known as papillomas in mice and used a conditional gene expression system to selectively label tumor cells with yellow fluorescent protein (YFP) (Driessens et al., 2012). YFP expression was dependent on genomic recombination induced by the drug tamoxifen, and the investigators restricted labeling to a handful of cells in each tumor by using low doses. These cells yielded fluorescent progeny, enabling assembly of clonal 'family trees' for individual founder cells as the papillomas grew.

The results proved surprising. " $80 \%$ of the labeled tumor cells seemed to disappear over time," says Blanpain. "The remaining $20 \%$ filled up a huge part of the tumor, and in every tumor we looked at there was always a huge chunk that came from a single cell." Collaborator

\section{GENOMICS}

\section{ÜBER-ACCURATE SEQUENCING}

Looking for agreement between Watson and Crick strands weeds out sequencing artifacts.

Michael Schmitt and Jesse Salk picked a tough problem to tackle. Working in the laboratory of Lawrence Loeb at the University of Washington, they wanted to identify the rate of low-frequency mutations in cancer cells. Salk, a medical resident, explains, "Cancer is a disease highly related to genetic heterogeneity; there is a lot of underlying variation that confers resistance to therapy."

The immediate obstacle they ran into was that high-throughput sequencing platforms have an error rate that is orders of magnitude too high to detect many naturally occurring variants. Illumina's HiSeq, for example, introduces one error in every 1,000 bases, whereas the somatic mutation rate is estimated to lie between 1 in $10^{8}$ and 1 in $10^{11}$.

Over the years, a number of other researchers have developed techniques to lower the error rate of high-throughput sequencing, either computationally-by improving the quality of base calls-or experimentally - by tagging all reads derived from the same original fragment and discarding any changes that are not seen in all reads with the same tag. These approaches can reduce the error rate to at best $10^{-5}$, but this is still not accurate enough for Schmitt and Salk's question.

Salk recalls being inspired by nature's ways of error correction. "There is reciprocally stored information in DNA," he says. "If there is an error in one strand that does not match the other side, it is recognized and corrected." They wanted to use the same approach to distinguish artifacts from real mutations and thus enlisted the help of Scott Kennedy, a postdoctoral fellow in the Loeb lab, to work out the computational and experimental problems.

The team's solution sounds deceptively simple but took some trial and error to bring to fruition. Each double-stranded DNA fragment is labeled on both ends with a duplex tag (a random, double-stranded, complementary adaptor) prior to single-strand sequencing. The 
Ben Simons, a physicist at Cambridge University, devised a model wherein papillomas contain a minority of rapidly dividing stem cells that self-renew but also give rise to slowly dividing 'progenitor' cells, which yield the differentiated cells that 'drop out' of tumor growth. By comparison, malignant skin tumors appeared to consist primarily of proliferative cells, suggesting that papilloma stem cells may provide the foundation for tumor progression.

Parada also found striking evidence for a central contribution of stem cells to tumorigenesis in an investigation of glioma, a brain malignancy (Chen et al., 2012). His team generated a mouse model of human glioma that incorporated a GFP transgene under the control of a neural-stem-cell-specific promoter. This transgene also expressed herpes simplex virus thymidine kinase, enabling the researchers to selectively kill these cells with the drug ganciclovir. Only a subset of tumor cells was GFP-positive, but these cells were critical to tumor growth. "When we gave them ganciclovir for extended periods of time, the tumors stop[ped] growing and the cells that were left over were not dividing," says Parada.

CSCs are also presumed to drive recurrence when therapy fails, and Parada investigated this possibility with temozolomide (TMZ), a standard chemotherapeutic agent. As in human patients, treatment in mice brought only temporary relief: the drug killed off much of the tumor but spared the population of labeled putative CSCs. These cells were relatively quiescent in the untreated tumor but leapt into action post-therapy. "All of the new cells that arose when TMZ was removed were derived from green-labeled cells," says Parada. Strikingly, sequential treatment with TMZ and ganciclovir effectively wiped out glioma growth.

These studies strongly suggest that stem cells may fuel tumor growth-and remain therapy resistant-in at least some cancers. "We're working on making a real link between the last 10 years of CSC research and this new line of research that we've begun," says Blanpain.

\section{Michael Eisenstein}

RESEARCH PAPERS

Chen, J. et al. A restricted cell population propagates glioblastoma growth after chemotherapy. Nature 488, 522-526 (2012).

Driessens, G. et al. Defining the mode of tumour growth by clonal analysis. Nature 488, 527-530 (2012).

resulting reads can then by grouped in two ways: by clustering single-strand sequences with the same tags to derive the single-strand consensus sequence-an approach similar to previous error-correction methods-or by matching the two complementary strands among single-strand consensus clusters to get the duplex consensus sequence. A mutation is only counted as real if it is supported by both strands.

In a first proof-of-principle experiment, the researchers sequenced a 7-kilobase bacteriophage genome with a mutation frequency of $3 \times 10^{-6}$. Their single-strand consensus sequences showed a mutation rate of $3.4 \times 10^{-5}$, indicating that $90 \%$ of all mutations seen were still artifacts. Their duplex consensus sequence, however, resulted in a rate of $2.5 \times 10^{-6}$, very close to the actual frequency.

The accuracy of duplex sequencing is impressive, but it requires considerable sequencing depth. To accurately quantify rare mutations, one needs to sequence an order of magnitude below the mutation frequency. For the M13 phage genome, this is doable; for an entire human genome, it would be very expensive. Instead, Schmitt and Kennedy applied duplex sequencing to the human mitochondrial genome, showing that the majority of mutations occurs at the origin of replication. Similarly, exomes or a select number of genes should be within reach of the method.

Loeb plans to use duplex sequencing on a few areas in a tumor genome. "So far the predominant mutations that are measured with sequencing are clonal," says Loeb, "but now I have the opportunity to measure [mutation frequency] subclonally and random[ly]. We can now look at cells with a great level of precision that has not been possible before."

Rare variants are of interest not only to the cancer field. Loeb and his colleagues also want to explore their effect in aging and neurodegeneration and seek rare variants in mixed microbial populations.

Now that the idea is developed, it's easy to plug it into existing pipelines and pursue rare variants in any system of choice.

Nicole Rusk 